\title{
Latency and Networking Aspects of Adaptive Modems over Slow Indoors Rayleigh Fading Channels
}

\author{
Jeff M. Torrance and Lajos Hanzo
}

\begin{abstract}
Adaptive modulation exploits the time-variant channel capacity fluctuation of fading channels using a range of different modem modes. Specifically, no information is transmitted when the instantaneous channel signal-to-noise ratio (SNR) is low, and, hence, during this period the data must be buffered, which results in delay or latency. When the instantaneous channel quality improves, 2-, 4-, 16-, and 64-level modem modes are invoked, which allows the transmission buffer to be emptied. It is shown that channel capacity gains are achieved at the cost of some latency penalty. The latency is quantified in this treatise and mitigated by frequency hopping or statistical multiplexing. The latency is increased when either the mobile speed or the channel SNR are reduced, since both of these result in prolonged low instantaneous SNR intervals. It is demonstrated that as a result of the proposed measures, typically more than 4-dB SNR reduction is achieved by the proposed adaptive modems in comparison to the conventional benchmark modems employed.
\end{abstract}

\section{INTRODUCTION}

$\mathbf{S}$ TEELE and Webb [1], [2] proposed adaptive differentially encoded, noncoherently detected star-constellation quadrature amplitude modulation [3] (Star-QAM) for exploiting the time-variant Shannonian channel capacity of fading channels. Their work has stimulated further pioneering work, in particular, by Kamio et al. at Osaka University and the Ministry of Post in Japan [4]-[10] as well as by Goldsmith et al. [11]-[17] at CalTech in the United States or by Pearce et al. [18] in the United Kingdom. Some of these contributions will be addressed in somewhat more depth at a later stage.

The principle of operation is to employ the most appropriate transmission scheme (TS) on a burst-by-burst basis in order to maintain a certain target bit error rate (BER) performance. The most appropriate TS is dependent upon the instantaneous signal-to-noise ratio (SNR), which varies as the channel experiences narrow-band fading. The TS that is used is selected

Manuscript received April 15, 1997; revised September 25, 1997. This work was supported by Motorola ECID, Swindon, U.K., European Community, Brussels, Belgium, Engineering and Physical Sciences Research Council, Swindon, U.K., and the Mobile Virtual Center of Excellence, U.K.

The authors are with the Department of Electronics and Computer Science, University of Southampton, SO17 1BJ Southampton, U.K. (e-mail: lh@ecs.soton.ac.uk; http://www-mobile.ecs.soton.ac.uk).

Publisher Item Identifier S 0018-9545(99)05784-9. as follows [19]:

$$
\mathrm{TS}= \begin{cases}\text { no transmission (Notx), } & \text { if } l_{1}>s^{2} / N \\ \text { BPSK, } & \text { if } l_{1} \leq s^{2} / N<l_{2} \\ \text { QPSK, } & \text { if } l_{2} \leq s^{2} / N<l_{3} \\ \text { square } 16 \text { point QAM, } & \text { if } l_{3} \leq s^{2} / N<l_{4} \\ \text { square } 64 \text { point QAM, } & \text { if } s^{2} / N \geq l_{4}\end{cases}
$$

where $s$ is the instantaneous signal level, $N$ is the average noise power, and $l_{1}, l_{2}, l_{3}$, and $l_{4}$, are the BER-dependent optimized switching SNR's. Time-division duplex (TDD) was proposed, in order to estimate the channel conditions on a slot by slot basis [20]. This requires uplink and downlink slots in the TDD frame to be adjacent for every user, rather than employing the conventional configuration, where the down- and uplink slots are in separate halves of the frame. Furthermore, bringing the corresponding duplex uplink and downlink slots closer to each other implies that now the time duration from the downlink to the next uplink transmission is extended and hence the channel correlation between the mobile reception and transmission is reduced. This problem can be mitigated by "passively" evaluating the received signal level of the TDD slot preceding the mobile's uplink slot. Following this "passive reception," uplink transmission takes place, which is the basis for the channel estimation for the downlink transmission, etc.

The upper bound BER and bit per symbol (BPS) channel efficiency performance of such a scheme in a Rayleigh fading channel was given in [19]. These adaptive schemes need a reliable transmission of the modulation-mode classifier, for which Otsuki et al. [7] proposed a Walsh-function-assisted signaling scheme to inform the receivers as to the modulation scheme employed, while in [21] an unequal protection five-phasor constellation was proposed for the same purpose, conveying the above-mentioned five modem modes used. Chua and Goldsmith [11] proposed the employment of concomitant variation of both the modulation scheme and the transmitted power, and in [12] they showed that there was a BER-dependent required power discrepancy between the their adaptive regime and the channel capacity. This corroborates the experience that the higher the acceptable BER, the better the system's capacity approximates the Shannonian channel capacity. They showed that using five-six different constellations it is possible to achieve a performance very close to that of the best possible system imposing no restrictions on the number of modulation constellations. 
TABLE I

Switching SNR's for Speech and Computer Data Systems Through A Rayleigh Channel, Shown in Instantaneous Channel SNR (dB) to Achieve Mean AND PeaK BER's of $1 \times 10^{-2}$ AND $1 \times 10^{-4}$

\begin{tabular}{l|c|c|c|c}
\hline Switching SNRs(dB) & $l_{1}$ & $l_{2}$ & $l_{3}$ & $l_{4}$ \\
\hline Peak-BER Speech (1\%) & 4.3 & 7.3 & 13.9 & 19.4 \\
Mean-Spcech $(1 \%)$ & 3.31 & 6.48 & 11.61 & 17.64 \\
Peak-BER Data $(0.01 \%)$ & 8.3 & 11.3 & 18.2 & 24.2 \\
Mean-BER Data $(0.01 \%)$ & 7.98 & 10.42 & 16.76 & 26.33 \\
\hline
\end{tabular}

In contrast to previous studies on modem spectral efficiency, where conventional fixed modems were used, in [3] the radio capacity of adaptive differentially detected noncoherent modems was studied by simulation using various radio cell constellations and a propagation prediction tool, while Alouini and Goldsmith in [16] derived theoretical expressions for the $\left[\mathrm{bits} / \mathrm{s} / \mathrm{Hz} / \mathrm{m}^{2}\right]$ capacity of adaptive modems. Further capacity-related work by the CalTech team was disseminated in References [13]-[16], while their contributions on adaptivecoded modulation were crystalized in [17]. Specifically, the simulation and theoretical results by Goldsmith and Chua showed that a 3-dB coding gain was achievable at a BER of $10^{-6}$ for a four-state trellis code and $4 \mathrm{~dB}$ by an eightstate trellis code in the context of the adaptive scheme over Rayleigh fading channels, while a 128 -state code performed within $5 \mathrm{~dB}$ of the Shannonian capacity limit. The benefits of automatic repeat request (ARQ) in adaptive modems were quantified by the Japanese teams from Osaka and Tokyo [10], while some of the latency aspects were addressed in [22].

It has also been shown [23] that Powell's optimization [24] may be employed to find the appropriate modulation switching SNR, in order to ensure that BER's of $1 \times 10^{-2}$ and $1 \times$ $10^{-4}$ are maintained for average channel SNR's in the range of $0-50 \mathrm{~dB}$ over a Rayleigh fading channel. For the lower delay, higher BER speech system and the lower BER, higher latency data system two different sets of optimum switching SNR's were designed, which maintained the above mean- and peak-BER's, respectively. The corresponding switching SNR's $l_{1}, l_{2}, l_{3}$, and $l_{4}$ are shown in Table I.

The average throughput $B$ of an adaptive modulation scheme, which is expressed in BPS, was derived for a Rayleigh fading channel in [19]

$$
\begin{aligned}
B= & 1 \cdot \int_{l_{1}}^{l_{2}} F(l, L) d l+2 \cdot \int_{l_{2}}^{l_{3}} F(l, L) d l \\
& +4 \cdot \int_{l_{3}}^{l_{4}} F(l, L) d l+6 \cdot \int_{l_{4}}^{\infty} F(l, L) d l
\end{aligned}
$$

where

$$
F(l, L)=\frac{1}{L} \cdot e^{-l / L}, \quad l \geq 0
$$

is the probability density function (PDF) of the received SNR $l=s^{2} / N$ and $L=S / N$ is the average received SNR, provided that the instantaneous signal amplitude $s$ is Rayleigh distributed, obeying [25]

$$
F(s, S)=\frac{2 s}{S} \cdot e^{-s^{2} / S}, \quad s \geq 0
$$

while $S$ is the average signal power. The individual terms in (2) correspond to the 1-, 2-, 4-, and 6-b/symb modem modes used between the corresponding thresholds of Table I. In this contribution, the actual average number of transmitted bits per symbol is kept below the BER-specific maximum value $B$ in order to be able to invoke more robust, but lower capacity and reduced-latency modem modes using the techniques to be highlighted during our further discourse.

Following a brief discussion on the latency problem and buffering in Section II our simulation model is described in Section III and the system's latency performance is characterized in Section IV. Then frequency hopping and statistical multiplexing are introduced in Sections V and VI, as two countermeasures exhibiting different properties, in order to mitigate the associated latency. Finally, Section VII provides a comparison with conventional fixed modulation schemes, before concluding in Section VIII.

\section{The LATENCY PROBlem}

An appropriate technique to manage the variable throughput intrinsic to adaptive modulation is to implement a buffer between the channel codec and the modulator. The buffer holds data when the channel conditions result in a lowthroughput, low-order modulation scheme being employed. When the channel conditions improve and high-order modulation schemes are employed, the buffer can be emptied. In order to make the system's operation plausible, as a stylized illustrative example, the instantaneous "fullness" of the buffer and the effective BPS performance $\bar{B}=\Xi \cdot B$ computed from (2) are shown in Fig. 1(a) as a function of the transmission frame index and average channel SNR, respectively. Our simulation model is the subject of the next section and hence at this early stage of our discussions it is premature to state quantitative system-performance details, apart from the fact that the buffer length has ramifications as to system latency.

Throughout our experiments we used normalized Doppler frequencies, in order to render our results more generically applicable to other systems. We note that normalization was carried out by multiplying the Doppler frequency $f_{D}$ expressed in hertz with the transmission frame duration of $4.615 \mathrm{~ms}$, a practical value used for example in the global system of mobile communications, known as the global system for mobile communication (GSM) [31], [32]. Lower normalized Doppler frequencies result in higher temporal channel correlation, which is exploited in order to estimate the channel quality and, therefore, to employ the most appropriate modulation scheme. Ironically, very low-Doppler channels result in prolonged fades that contribute to the adaptive modulation's latency.

For given average channel conditions the average BPS performance of an adaptive modulation scheme in a Rayleigh fading channel is characterized by (2). In practice, if a buffer is to be employed, the average throughput must be less than this performance limit, in order to prevent a bufferlength buildup, which would result in increased latency in interactive speech or video communications. Therefore, it is assumed that the average number of transmitted bits per symbol period is given by $\bar{B} \leq B$, allowing us to define the 


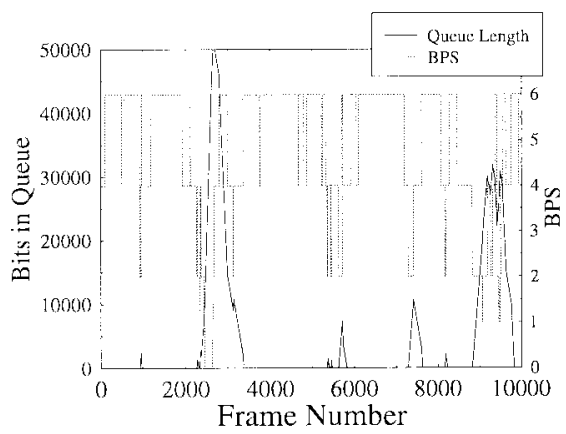

(a)

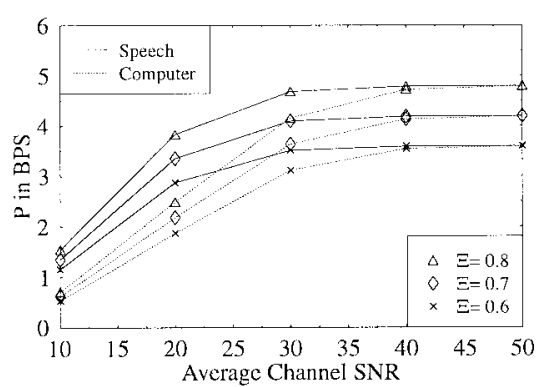

(b)

Fig. 1. Instantaneous buffer length, instantaneous throughput, and BPS performance for adaptive speech and computer data schemes, $\Xi$ values of $0.6,0.7$, and 0.8 , and for a range of average channel SNR's: (a) instantaneous length of the buffer and the instantaneous BPS throughput and (b) effective BPS performance $\bar{B}=\Xi \cdot B$ of the adaptive speech and computer data schemes for $\Xi$ values of $0.6,0.7$, and 0.8 , and for a range of average channel SNR's computed from (2).

"relative channel utilization factor" $\Xi=\bar{B} / B$. Explicitly, in order to prevent a buffer overflow and high speech or video latency, the average number of bits transmitted per symbol is kept below $B$, therefore, $\Xi<1$. The buffer will introduce latency to the transmission and this latency will depend upon the fading frequency, the modulation switching SNR's, the average channel SNR and $\Xi$. The average BPS performance of an adaptive, $1 \%$ mean BER speech, and a $0.01 \%$ mean BER computer data scheme for $\Xi$ values of $0.6,0.7$, and 0.8 is shown for a range of average channel SNR's in Fig. 1(b).

\section{Simulation Model}

For the modeling of the Rayleigh fading channel, two approaches have been considered. Jakes [33] describes and Casas et al. [34] implement in hardware a technique referred to as "sum of sinusoids" for simulating Rayleigh fading using the summation of several low-frequency sinusoids with regularly spaced phase differences. An alternative approach performs complex addition of two band-limited quadrature additive white Gaussian noise (AWGN) sources, a solution advocated for example by Arredondo et al. [35] and Goubran et al. [36]. We carried out a rigorous $\chi^{2}$ Rayleigh-distribution hypothesis testing [37] for the two methods and for a variety of reasons the latter quadrature noise source method was preferred.

A convenient approach to understanding how the above parameters will affect the latency of an adaptive modulation scheme is to describe the transmission mode switching scheme with a Markov model [26]. This approach is appropriate for modeling the throughput of adaptive modulation, because the modulation scheme employed is only dependent upon which of the switching SNR's the instantaneous SNR falls between. By contrast, the BER depends upon the actual value of the instantaneous SNR.

Adaptive modulation is applicable to a range of system environments, such as that of the digital European cordless telephone (DECT) [27], where the frame length is 10 $\mathrm{ms}$, to an advanced time-division multiple-access (ATDMA) environment [28] with a 5-ms frame length or to the forthcoming universal mobile telecommunications system's (UMTS) indoor-specific environment [29], [30], which has a frame
TABLE II

MaXimum Normalized Doppler Frequencies for Which the Wang-Moayeri [38] Markov Model Transition Probabilities Show Good Correspondence with the AdAPTIVe Modem Mode Transition Probabilities Evaluated by Simulation

\begin{tabular}{l|c|c|c|c}
\hline & $20 \mathrm{~dB}$ & $30 \mathrm{~dB}$ & $40 \mathrm{~dB}$ & $50 \mathrm{~dB}$ \\
\hline Speech & $2.52 \times 10^{-2}$ & $7.41 \times 10^{-3}$ & $2.22 \times 10^{-3}$ & $7.41 \times 10^{-4}$ \\
Data & $3.19 \times 10^{-2}$ & $9.64 \times 10^{-3}$ & $2.97 \times 10^{-3}$ & $7.41 \times 10^{-4}$ \\
\hline
\end{tabular}

length of $4.615 \mathrm{~ms}$. This frame length is also identical to that of the GSM system [31], [32].

Accordingly, every $4.615 \mathrm{~ms}$ the TS to be employed by a particular user was reevaluated. The normalized Doppler frequency of the fading, again, was computed with respect to the transmission frame rate, rather than to the more conventionally used symbol duration, and this determined how often actually the employed TS varied. Markov techniques were used to model the TS that would be employed. Transition probabilities between "adjacent" TS schemes, such as BPSK and 4QAM may be evaluated analytically using the approach of Wang and Moayeri [38] or by simulation. However, simulations showed that the probability of nonadjacent transitions, such as a BPSK frame followed by a 64-QAM frame were not negligible for the system parameters of interest, in particular for more rapidly fluctuating higher Doppler frequencies. Hence, we decided to determine the maximum Doppler frequency up which the Wang-Moayeri [38] model is applicable to our adaptive regime using $\chi^{2}$ testing [24], [37].

The normalized Doppler frequencies, where the $\chi^{2}$ confidence levels became unacceptable are tabulated in Table II. Inspection of this table reveals that, for both sets of switching SNR's, the maximum normalized Doppler frequency, for which the Wang-Moayeri model is applicable to our problem increases with reduced average channel SNR. Due to lack of space this plausible phenomenon and a range of other associated issues are left for the interested reader to interpret in detail.

Considering the 4.615-ms frame length, if the Wang-Moayeri model is to be invoked for evaluation of the latency effects of adaptive modulation, for average channel SNR's up to $50 \mathrm{~dB}$ and for both computer data and 


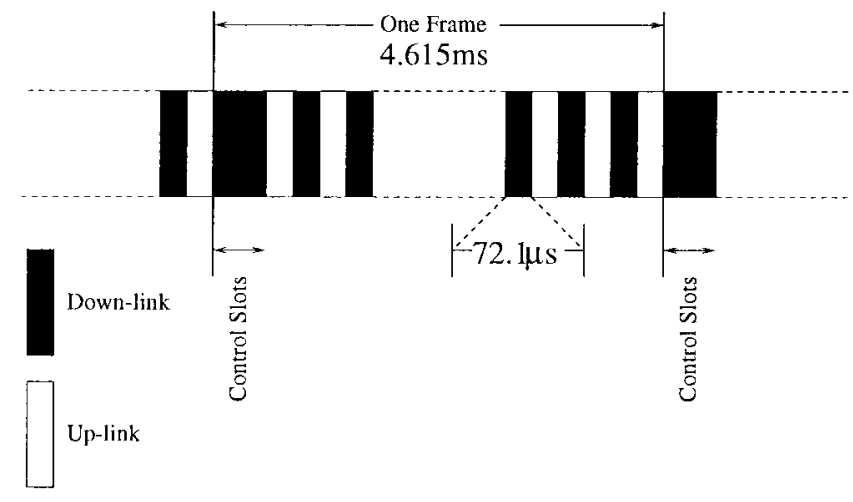

Fig. 2. TDD/TDMA frame for employment of statistical multiplexing evaluation of latency in FRAMES TDD mode.

speech models, the maximum Doppler frequency that can be successfully modeled is $3.19 \times 10^{-2} / 4.615 \times 10^{-3}=5.5 \mathrm{~Hz}$. Assuming the burst structure proposed for UMTS by the panEuropean FRAMES consortium [30], under the auspices of the advanced communications technologies and services (ACTS) program, and a carrier frequency of $2 \mathrm{GHz}$, the maximum normalized Doppler frequency of $5.5 \mathrm{~Hz}$ corresponds to a pedestrian velocity of $0.82 \mathrm{~ms}^{-1}$, which is fairly low. Hence, for the sake of generality, the Markov transition probabilities used to evaluate the latency of our adaptive modem were generated by simulation. Furthermore, these simulated transition probabilities include the finite probability of transition to and from "nonadjacent" transmission modes of the adaptive regime. The Markov transition probabilities of our adaptive modem were evaluated by simulation for average channel SNR's of 10, 20, 30, 40, and $50 \mathrm{~dB}$, and for normalized Doppler frequencies of 0.0042, 0.025, 0.054, 0.079 , and 0.133 over a Rayleigh channel, using both the peak and mean BER $1 \%$ and $0.01 \%$ (speech and computer data system) switching SNR's of Table I.

In order to stipulate the system parameters, the general issue of latency in adaptive modulation is considered complying with the FRAMES consortium's approach to UMTS, which was highlighted by Ryck et al. [30]. The current FRAMES proposal in the $2-\mathrm{GHz}$ band includes a time division duplex (TDD) option with a 4.615-ms frame duration, up to 64 slots/frame for $2-\mathrm{GHz}$ transmission. Including adaptive modulation in this framework results in a temporal difference of $72 \mu$ s between channel quality estimation and transmission, assuming that uplink and downlink slots consecutively follow each other, as portrayed in Fig 2, and "passive reception" of the preceding time-slot's signal is invoked, in order to infer an estimate of the uplink channel's quality. This figure will be discussed in more depth during our further discourse. At a vehicular speed of $5 \mathrm{~ms}^{-1}, 72 \mu$ s corresponds to $360 \mu \mathrm{m}$ or to a minute fraction of about $0.5 \%$ of the carrier wavelength. Therefore, it is assumed that the fading envelope does not vary significantly between estimation and transmission.

Accordingly, every 4.615-ms TDD frame the transmission scheme to be employed by a particular user will be reevaluated. The Doppler frequency, normalized to the transmission frame rate, will determine how often the employed transmission scheme will actually vary. The modulation mode transitions for a particular user were characterized by a Markov model, where the transition probabilities were evaluated by simulation for the sake of maintaining validity at higher Doppler frequencies, as argued before. Our performance results are related to a propagation frequency of $2 \mathrm{GHz}$, to a frame duration of 4.615 $\mathrm{ms}$, and to normalized Doppler frequencies of $0.0042,0.025$, $0.054,0.079$, and 0.133 , which correspond to mobile velocities of $0.136,0.812,1.75,2.57$, and $4.32 \mathrm{~ms}^{-1}$.

\section{Single-Slot PERFORMANCE}

The initial evaluation of the latency considers a single user, who is allocated a single uplink slot within the TDMA/TDD frame of Fig. 2, where the uplink slot is immediately followed by the downlink slot for the same user, noting that the associated hardware ramifications are beyond the scope of this paper. It is assumed that the desired throughput for the uplinks and downlinks is the same, what we refer to as "balanced TDD." Furthermore, because the channel is assumed to be reciprocal, the latency experienced by both the uplink and downlink transmissions is the same. Every user may employ "no transmission," BPSK, QPSK, square 16, or 64 QAM in the slot allocated to them. The same modulation scheme must be employed over the full length of the slot. It was also assumed that the buffer was initially empty.

The delay for a single user, that is allocated a single slot, transmitting over a Rayleigh fading channel was evaluated using the configuration of Fig. 2 cumulative distribution functions (CDF's) of the number of frames, by which each bit was delayed, were evaluated for $2 \times 10^{6}$ frames, with $\Xi$ of 0.6 , 0.7 , and 0.8 , average channel SNR's of $10,20,30,40$, and 50 $\mathrm{dB}$, normalized Doppler frequencies of $0.0042,0.025,0.054$, 0.079 , and 0.133 , and for both the BER $=1$ optimized speech and the $\mathrm{BER}=0.01$ optimized computer data transmission switching SNR's of Table I in the absence of interference. It should be noted that during this evaluation of latency there was no limit imposed upon the buffer length. However, in Section VII-B, where adaptive modulation is compared to fixed schemes, the buffer is limited to the maximum allowed speech-specific and memory-constrained data latency. Fig. 3(a) and (b) are examples of such delay CDF's, expressed in terms of the number of 4.615 -ms frames for $\Xi=0.6$, for various normalized Doppler frequencies and for our speech and data schemes, respectively.

Observe in Fig. 3 that for both speech and data transmission the CDF reaches unity less rapidly, as the Doppler frequency is reduced, implying an increased relative frequency of longer buffer buildup. Hence, as expected, the average delay increases with lower normalized Doppler frequencies. Furthermore, due to its more stringent BER, the computer data system results in longer delays than the speech system. The effect of a lower normalized Doppler frequency is that it prevents the transmission scheme from changing very frequently. Hence, there are relatively long periods, where the instantaneous transmission capacity is higher than the input rate, followed by relatively long periods of the reverse situation. This is what results in bursts of latency. At higher normalized Doppler 


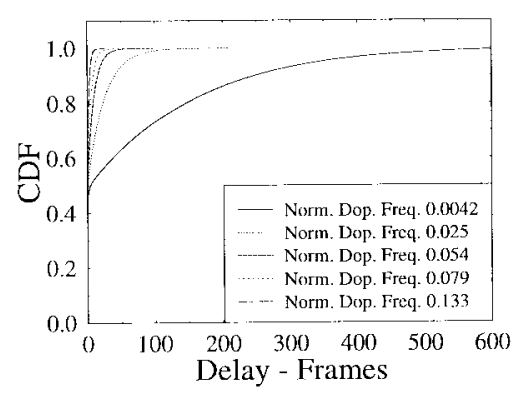

(a)

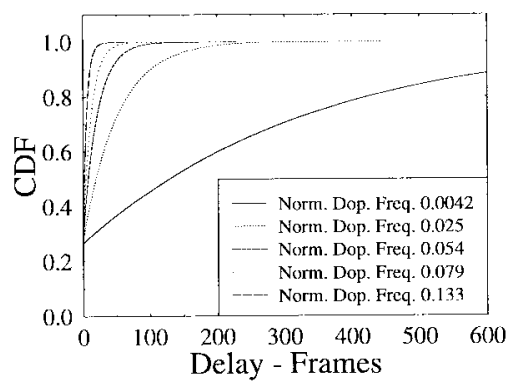

(b)

Fig. 3. CDF of delay expressed in terms of 4.615-ms frames for (a) speech and (b) computer data transmission with $\Xi$ of 0.6 and various normalized Doppler frequencies.

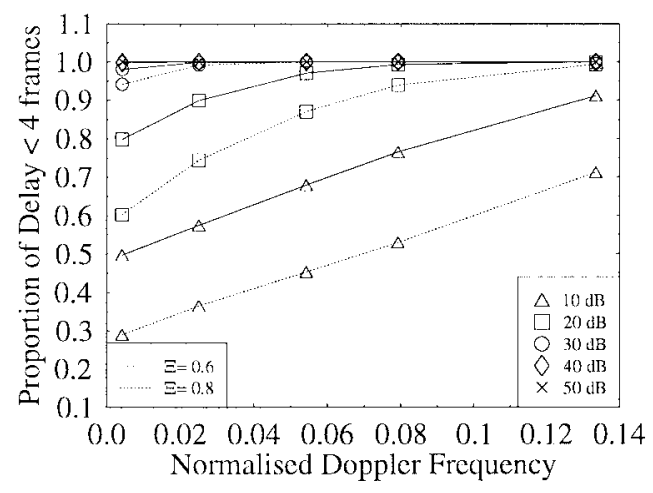

(a)

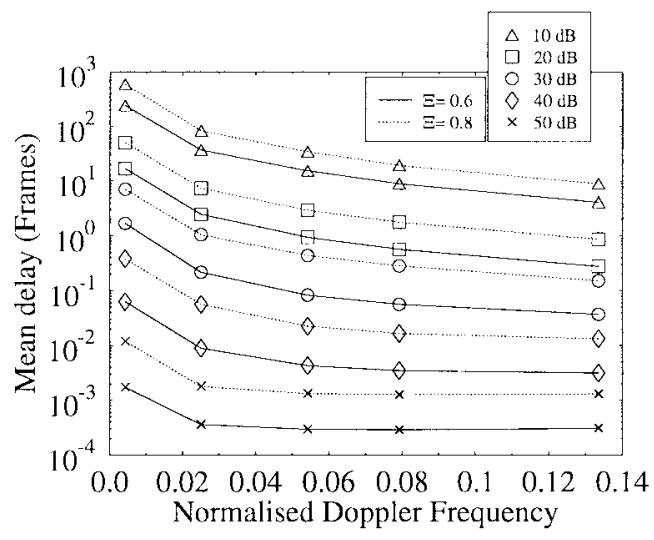

(b)

Fig. 4. Delay over a Rayleigh fading channel as a function of the normalized Doppler frequency for $\Xi=0.6$ and 0.8 , assuming average channels SNR's of 10,20,30,40, and $50 \mathrm{~dB}$ : (a) proportion of transmitted bits in speech system experiencing four or fewer frames latency and (b) mean delay of bits expressed in terms of the number of frames for the computer data system.

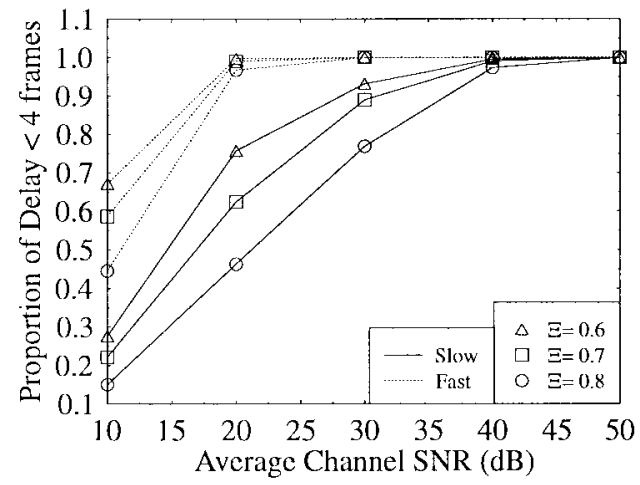

(a)

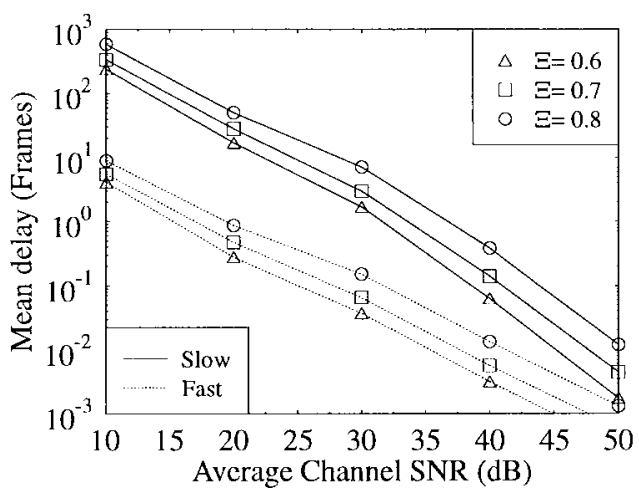

(b)

Fig. 5. Delay over a Rayleigh fading channel as a function of the average channel SNR for $\Xi$ values of 0.6 , 0.7, and 0.8 and for normalized Doppler frequencies of 0.004170 (slow) and 0.133427 (fast): (a) proportion of transmitted bits in speech system experiencing four or fewer frames latency and (b) mean delay of bits expressed in terms of the number of frames for the computer data system.

frequencies, the transmission scheme changes more frequently and the latency is reduced.

For the speech system a maximum of four-frames latency introduced by the modem was considered acceptable, which corresponded to less than $20 \mathrm{~ms}$, or one typical linear predictive analysis speech coding frame duration [39]. The delay CDF's of Fig. 3 were processed in order to determine the proportion of bits that were transmitted within the acceptable four-frame latency. For the computer data system no maximum delay was imposed, instead, the mean delay was considered. Figs. 4(a) and (b) and 5(a) and (b) illustrate these measures of latency for both systems as a function of the normalized Doppler frequency and average channel SNR, which are detailed below. 
Specifically, Fig. 4(a) shows that an increased normalized Doppler frequency reduces the latency. This is particularly conspicuous when the average channel SNR is low, manifesting itself in a near-constant CDF gradient tending toward unity. For high average SNR's the Doppler frequency is less critical, which can be confirmed for example by comparing the curves associated with the average channel SNR's of 10 and $30 \mathrm{~dB}$. This improvement results from the reduced number of consecutive "no transmission" frames. At $30 \mathrm{~dB}$, the probability of "no transmission" is very low and, therefore, even a highly correlated, slowly fading channel results in only a few adjacent "no transmission" frames. Although the full set of Markovian transition probabilities are not included for reasons of space economy, by considering these transition probabilities this tendency can be confirmed. For example, while in a slow fading channel exhibiting a normalized Doppler frequency of 0.004170 and a rather low 10-dB average channel SNR, we found that the probability of "no transmission" was 0.192 and the probability of remaining in "no transmission," given that this is the current transmission scheme was found to be 0.980 . By contrast, at the significantly higher $30-\mathrm{dB}$ SNR these probabilities became 0.0021 and 0.775 , respectively, which reflects the reduction in delay inferred from Fig. 5.

For average channel SNR's in excess of $20 \mathrm{~dB}$, the latency performance shown in Fig. 4(a) is nearly always acceptable for speech systems, irrespective of the value of the "relative channel utilization factor" $\Xi$ when the normalized Doppler frequency is higher than 0.025. However, at lower average channel SNR's, the $\Xi$ value has a greater bearing upon the latency. Therefore, in principle the speech codec could be reconfigured depending upon the average channel SNR, as proposed in [40]. However, whereas in [40], the speech codec was reconfigured in order to transmit the lower rate speech signal using a more robust, but lower capacity modem mode, in our current system it would be reconfigured in order to reduce the latency inflicted by the lower average channel SNR experienced.

Observing Fig. 4(b) now reveals that the mean delay for the computer data system is also reduced, as the normalized Doppler frequency increases. It is interesting to note that for all $\Xi$ values and for all average channel SNR's the shape of the curves in the figure is similar. Bearing in mind the logarithmic nature of the $y$ axis, this figure again shows that the increased decorrelation of higher Doppler-frequency channels has greater effect upon the latency of systems under low average channel SNR conditions. Furthermore, most of the delay-reduction is observed due to the initial increases in normalized Doppler frequency, whereas for values in excess of about 0.02 the curves become more flat.

Fig. 5(a) shows how the latency performance of our fastest and slowest experimental fading channels, associated with the Doppler frequencies of 0.004170 and 0.133427 , respectively, converge at high average channel SNR's, irrespective of the $\Xi$ values. This is because the instantaneous channel SNR is virtually always greater than $l_{4}$ and, therefore, nearly always square 64 QAM is employed, irrespective of the normalized Doppler frequency. At 20-dB average channel SNR, the latency is almost independent of $\Xi$ for the faster fading channel, while for the lower fading rate there is nearly a factor of two performance difference between $\Xi$ values of 0.6 and 0.8 .

Fig. 5(b) shows the convergence in latency performance for both fading rates at high average channel SNR's. The figure also suggests that an increased average channel SNR results in more significant benefits in terms of latency performance for slower fading by exhibiting a steeper gradient. However, the increased additional average channel SNR also increases the average BPS performance $(\bar{B})$ as shown in Fig. 1(b). In this context, increasing the average channel SNR reduces the latency, while increasing the throughput.

In summary of our previous discourse, the latency ramifications of adaptive modulation in a variety of scenarios have been considered. The following two sections consider techniques mitigating the latency introduced by adaptive modulation.

\section{FREQUENCY HOPPING}

The results above show that reduced correlation in the fading channel reduces latency in an adaptive modulation scenario, although it degrades the quality of the channel estimation due to the more rapidly evolving fading envelope. The correlation may be reduced by including frequency hopping into the system. This has no adverse effects upon the channel estimation when it is performed using the previously mentioned "passive reception," where the estimated TDD uplink timeslot's channel quality is inferred from passively evaluating the quality of the preceding downlink timeslot. The other advantage of decorrelating the fading is that the PDF of the instantaneous BER will have a lower variance and, hence, will be more concentrated around the average BER experienced by the source-coded frames, since the amount of time spent in a deep fade is statistically speaking limited to a single burst, rather than inflicting long strings of high-BER frames. This vindicates the decision to use the mean BER switching SNR's, from Table I for these experiments rather than the peak BER switching SNR's.

The proposed frequency hopping scheme was simulated assuming $M$ independent fading channels, with a frequency hop every TDD frame. All of the previous experiments were repeated employing $M=2,3,5$, and 10 fading channels. Fig. 6(a) and (b) shows the effect of $M$ independent frequency-hopped channels upon the latency of the speech and computer data transmission systems, for 10 and $20-\mathrm{dB}$ average channel SNR's, $\Xi$ values of 0.6 and 0.8 , as well as for normalized Doppler frequencies of 0.004170 (slow) and 0.133427 (fast). Note in the figure that bold markers correspond to slow and hollow ones to fast fading. These figures reveal that significant delay reductions may be achieved by introducing frequency hopping to an adaptive modulation scheme. Ten hopping frequencies allow virtually all bits under all circumstances in the speech system to exhibit less than four-frames latency. Observe in Fig. 6(b) for the computer data system that all mean delays are reduced below 20 frames, which is equivalent to $92 \mathrm{~ms}$. The frequency hopping rate of $1 / 4.615 \mathrm{~ms} \approx 217 \mathrm{hops} / \mathrm{s}$ is identical to that of the GSM system and at this slow hopping rate the synthesizer design does not constitute a serious challenge. In the next section, 


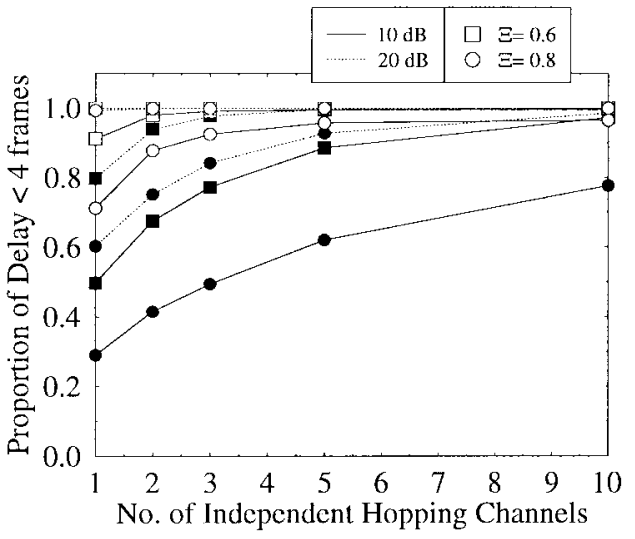

(a)

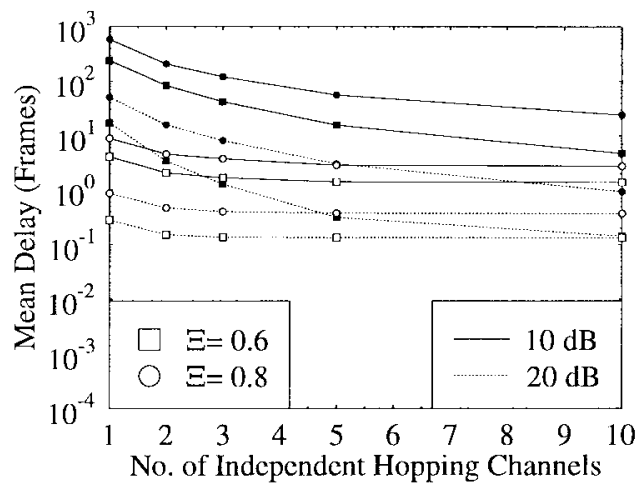

(b)

Fig. 6. Adaptive transceiver delay over independent frequency-hopped channels for 10- and 20-dB average channel SNR's, $\Xi$ values of 0.6 and 0.8 , and normalized Doppler frequencies of 0.004170 (slow) and 0.133427 (fast). Bold markers correspond to slow hollow ones to fast fading: (a) proportion of transmitted bits in speech system experiencing four or fewer frames latency and (b) mean delay of bits expressed in terms of the number of frames for the computer data transmission system.

TABLE III

Total Number of UplinK AND DOWNLINK SLOTS IN a Frame for Balance TDD with Statistical Multiplexing

\begin{tabular}{l|c}
\hline Modulation Scheme & Slots Occupied \\
\hline No Transmission & 2 \\
BPSK & 8 \\
QPSK & 6 \\
Square 16 QAM & 4 \\
Square 64 QAM & 2 \\
\hline
\end{tabular}

let us now consider an alternative technique of mitigating the latency effects, namely statistical multiplexing.

\section{Statistical MultiPleXing}

\section{A. Background}

Pearce et al. [18] considered allowing users to occupy a varying number of slots on the basis of the traffic, the modulation scheme, and path loss that is experienced. Williams et al. [41] considered employing packet reservation multiple access (PRMA) combined with different modulation schemes in order to accommodate different channel qualities associated with different average channel SNR's and interference loads across a cell. These ideas are based on the bursty nature of speech data, assuming that a speech codec with voice activity detection (VAD) [32] is used, as proposed for example by Goodman et al., Nanda et al., and Wong in [42]-[45], respectively. Here a different statistical multiplexing scheme is exploited in order to mitigate the delay in an adaptive modulation scheme. It is only considered for the speech switching SNR's, because the computer data is less dependent upon system latency. We note, however, that invoking this technique implies both an increased system complexity and the inclusion of a packet header overhead.

In our proposed statistical multiplexing scheme, the number of slots allocated to a user is now adaptively controlled in order to compensate for the different BPS channel capacity of the various modem modes. Table III shows the number of TDD slots allocated to the various modem modes. As the channel conditions deteriorate, the modulation order is reduced and, therefore, the number of slots per frame is increased. These slot-demand increases in a TDD frame are statistically independent of each other for all users and, hence, assuming a sufficient number of users, the variation in demand is averaged. When the channel becomes so poor that transmissions will result in an unacceptable error rate, that is, the modem is in the "no transmission" mode, no slots need to be occupied for transmission, however, one uplink and one downlink slot is reserved in order to assist in channel estimation. Here, we refrain from detailing the corresponding base station and mobile station operations, which are summarized in more depth in the Appendix.

\section{B. Statistical Multiplexing Performance}

The performance of the proposed statistical multiplexing scheme was evaluated for $5,10,15,20,25$, and 30 users and 20-, 30-, 40-, and 50-dB average channel SNR's. In all cases, we employed $\Xi=0.8$, the peak BER switching SNR's were used and for each simulation all users were assumed to be experiencing the same average channel SNR. The performance was only evaluated for the $1 \%$ BER adaptive speech scheme and results were obtained for normalized Doppler frequencies of $0.0042,0.025,0.054,0.079$, and 0.133 , which correspond to mobile velocities of $0.136,0.812,1.75,2.57$, and $4.32 \mathrm{~ms}^{-1}$.

Tables IV and V summarize the number of users accommodated on a single adaptive TDD carrier using the structure of Fig. 11, with a $99 \%$ and $95 \%$ probability of a four or fewer frame delay, corresponding to a maximum of about $20 \mathrm{~ms}$. When all users experience $40-$ or $50-\mathrm{dB}$ average channel SNR, 30 "balanced duplex" users can be accommodated, assuming the FRAMES configuration. There is a limit of 31 duplexed users because the proposed statistical multiplexing algorithm maintains one pair of slots for each user, even if they are not transmitting, as was shown in Table III. Furthermore, one pair of slots are used for sending the broadcast control slotstatus information for the statistical multiplexing, as seen in 
TABLE IV

Number of Users That Can Be Accommodated in Rayleigh Fading When All Users Experience the Same Average Channel SNR for a Range of Normalized Doppler Frequencies Using Statistical Multiplexing with 99\% of Frames Being Delayed less than the Duration of Four Frames. The Contention Permission Probability is $p$

\begin{tabular}{l|c|c|c|c}
\hline SNR & $50 \mathrm{~dB}$ & $40 \mathrm{~dB}$ & $30 \mathrm{~dB}$ & $20 \mathrm{~dB}$ \\
\hline Users & 30 users for all $p$. & 30 users for all $p$. & 25 users for all $p$ & 15 users for \\
Accom- & & & 30 users for & $f_{d} T \geq 0.025$ and \\
modated & & & $\begin{array}{c}f_{d} T \geq 0.025 \text { and } \\
p \neq 0.1,0.7,0.8\end{array}$ & $\begin{array}{c}p \neq 0.1,0.2,0.6 \\
\end{array}$ \\
& & & or 0.9. & \\
& & & & \\
\hline
\end{tabular}

TABLE V

Number of Users That Can Be Accommodated in Rayleigh Fading When All Users Experience the Same Average Channel SNR for a Range of Normalized Doppler Frequencies Using Statistical MultipleXing with 95\% of Frames Being Delayed Less Than the Duration of Four Frames. The Contention Permission Probability is $p$

\begin{tabular}{l|c|c|c|c}
\hline SNR & $50 \mathrm{~dB}$ & $40 \mathrm{~dB}$ & $30 \mathrm{~dB}$ & $20 \mathrm{~dB}$ \\
\hline Users & 30 users for all $p$. & 30 users for all $p$. & 30 users for all $p$. & 15 or 20 users \\
Accom- \\
modated
\end{tabular}

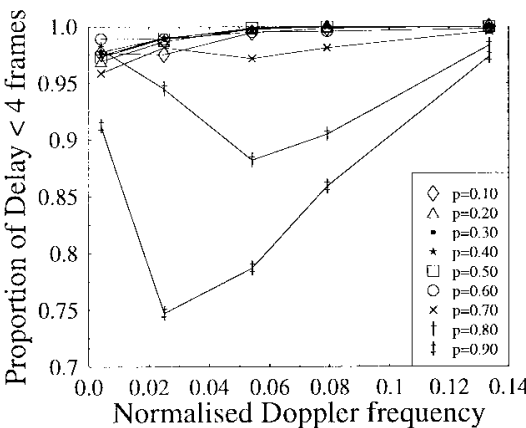

(a)

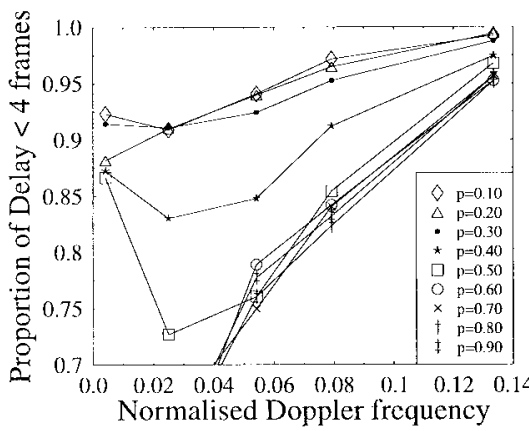

(c)

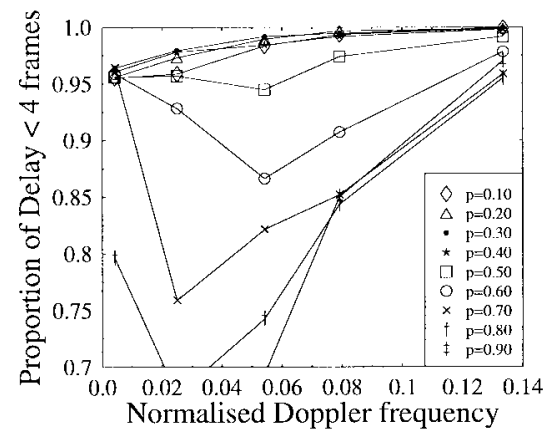

(b)

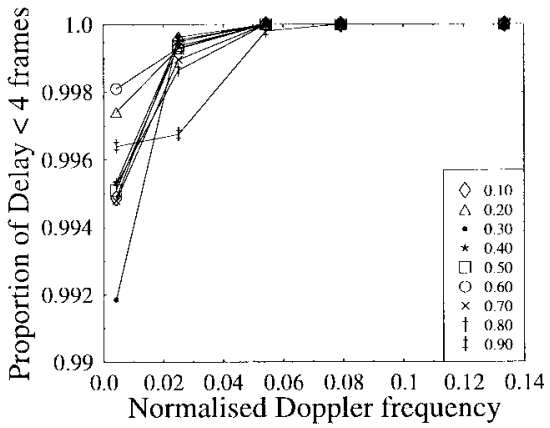

(d)

Fig. 7. Proportion of speech frames delayed more than four frames for contention permission probabilities of $p=0.1,0.2, \ldots 0.9$, for a $\Xi$ value of 0.8 with various average channel SNR's and a range of normalized Doppler frequencies: (a) 15 users with 20-dB average channel SNR, (b) 20 users with 20-dB average channel SNR, (c) 25 users with 20 -dB average channel SNR, and (d) 25 users with 30-dB average channel SNR.

Fig. 2. Employing two rather than one control slots allows us to accommodate strong channel coding and to exploit the otherwise unused 32nd uplink slot duration.

The performance of the statistical multiplexing scheme is considered in more detail at SNR's of 20 and $30 \mathrm{~dB}$ in Fig. 7. Fig. 7(a) shows that a high-contention permission probability $p$ increases the delay, since there are too many packet collisions and renewed contentions. However, a large $p$ has greater negative effect at 0.025 normalized Doppler frequency than at 0.0042 . This is because at very low normalized Doppler frequencies the modulation scheme employed on a particular base-station to mobile-station link changes very infrequently. Therefore, the modem mode does not change frequently and hence, extra slots are rarely required and consequently contention rarely occurs. However, as the normalized Doppler frequency increases, slots in the frame are exchanged between users more frequently and the probability of contention increases. This increase in contention is superimposed on the 
TABLE VI

Summary of Burst Configurations of Fixed Modulation Schemes to Be Used for Comparison with Adaptive Modulation

\begin{tabular}{c|c|c|c|c|c|c}
\hline $\begin{array}{c}\text { Fixed } \\
\text { Scheme }\end{array}$ & $\begin{array}{c}\text { Bits Per } \\
\text { Slot }\end{array}$ & $\begin{array}{c}\text { Slots Per } \\
\text { Frame }\end{array}$ & $\begin{array}{c}\text { Bits Per } \\
\text { Frame }\end{array}$ & $\begin{array}{c}\text { Block } \\
\text { Code }\end{array}$ & $\begin{array}{c}\text { Codewords } \\
\text { Per Frame }\end{array}$ & $\begin{array}{c}\text { Info Bits } \\
\text { Per Frame }\end{array}$ \\
\hline BPSK & 64 & 6 & 384 & $127,64,10$ & 3 & 192 \\
QPSK & 128 & 3 & 384 & $127,64,10$ & 3 & 192 \\
QAM 16 & 256 & 2 & 512 & $127,50,13$ & 4 & 200 \\
QAM 64 & 384 & 1 & 384 & $127,64,10$ & 3 & 192 \\
\hline
\end{tabular}

effect that as the normalized Doppler frequency increases, the adaptive scheme's throughput varies less from the average throughput and therefore fewer symbols spend a long time in the buffer.

Comparing Fig. 7(a) with (b) shows how the probability of unacceptable delay increases as the number of users is increased from 15 to 20 , by $33 \%$. The delay becomes worse at all normalized Doppler frequencies and $p$ becomes a more critical parameter after this increase of the number of users. A further increase from 20 to 25, by the same number of users is illustrated by Fig. 7(c). Again, the delay performance becomes worse at all normalized Doppler frequencies. Also shown in this figure is, assuming 20-dB average channel SNR and 25 users that the contention at very low normalized Doppler frequencies results in catastrophic delays if $p \geq 0.60$. From Fig. 7(a) to (c), it was decided to use a permission probability of 0.3. Fig. 7(d) shows the effect of increasing the SNR from 20 to $30 \mathrm{~dB}$ with respect to Fig. 7(c).

\section{COMPARISON OF FIXED AND AdAPTIVE ModeMS UsING BLOCK CODING}

\section{A. Fixed Schemes}

In this section, adaptive and fixed modulation techniques are compared in terms of the number of duplex users they can support on a single carrier frequency at practical indoors velocities. Block coding is employed and an indoor propagation environment is assumed. The data frame duration is again, $4.615 \mathrm{~ms}$ and the frames contain 64 slots, each with a useful payload of 64 data symbols and due to the overhead of ramp, guard, and training symbols, the gross symbol rate is 1.3 $\mathrm{MBd}$. This rate can be accommodated in the 1.6-MHz TDD bandwidth we assumed, while using a Nyquist rolloff factor of 0.35 and allowing for some adjacent channel interference. A continuous balanced-duplex TDD service is assumed with a useful payload of $192 \mathrm{~b} / 4.615 \mathrm{~ms}=41.6 \mathrm{~Kb} / \mathrm{s}$, which becomes $200 \mathrm{~b} / 4.615 \mathrm{~ms}$ for the 16 QAM mode of Table VI. The intended application for such a transmission scheme is a mixed video and speech service, for example, speech encoded using a codec of the type standardized by the ITU as G.729 [46] and synchronized video encoded using a codec of the type that is also standardized by the ITU as H.263 [47]. The tolerable transmission frame error rate (FER) is in general dependent on the source codecs, channel codecs, and other transceiver features, but typically it is expected to be below $1 \%$. We assume that the affordable transmission delay is 30 $\mathrm{ms}$. This is based upon a maximum of $60-\mathrm{ms}$ system delay (GSM [31], [32] has a speech delay of $57.5 \mathrm{~ms}$ ), where for example 10-ms delay is induced by the G.729 source encoding
TABLE VII

Average Channel SNR (dB) Required to Obtain 1\% FER When Using the Fixed Modulation Schemes Described in Table Vi Under Slow $\left(0.136 \mathrm{~ms}^{-1}\right)$ AND FAST $\left(4.32 \mathrm{~ms}^{-1}\right)$ RAYLEIGH FADING CONDITIONS

\begin{tabular}{c|c|c|c|c}
\hline & \multicolumn{2}{|c|}{ SNR (Slow) } & \multicolumn{2}{c}{ SNR (Fast) } \\
\hline $\begin{array}{c}\text { Modulation } \\
\text { Scheme }\end{array}$ & $\begin{array}{c}\text { Not } \\
\text { Interleaved }\end{array}$ & Interleaved & $\begin{array}{c}\text { Not } \\
\text { Interleaved }\end{array}$ & Interleaved \\
\hline BPSK & 20.0 & 20.0 & 20.3 & 17.7 \\
QPSK & 23.0 & 23.0 & 23.1 & 20.7 \\
QAM 16 & 27.7 & 27.7 & 28.0 & 23.7 \\
QAM 64 & 33.9 & 33.9 & 34.2 & 30.5 \\
\hline
\end{tabular}

and decoding, and a further $10 \mathrm{~ms}$ is reserved for the so-called processing delay, yielding a total of $30-\mathrm{ms}$ speech coding delay. The computational processing time could potentially be reduced, however, a faster processor would be required and there would be a consequential increase in power requirements. Interference is not considered at this stage.

We evaluated the performance of conventional fixed BPSK, QPSK, square 16, and 64 QAM separately over Rayleigh fading channels. The throughput was $41.6 \mathrm{~Kb} / \mathrm{s}$ for all schemes and, therefore, these schemes required six, three, two, and one slots per user, respectively. For square 64 QAM, QPSK, and BPSK, this results in $384 \mathrm{~b} /$ frame, and for square 16 QAM, $512 \mathrm{~b} /$ frame. The $384 \mathrm{~b} /$ frame, for square 64 QAM, QPSK, and BPSK, consists of three Bose-Chaudhuri-Hocquenghem $(\mathrm{BCH})(127,64,10)$ block codes [48], where three transmitted bits will be wasted. The square 16 QAM will use four $\operatorname{BCH}(127,50,13)$ block-coded word and waste four bits. The coding rates are close to 0.5 ; half-rate codes are widely used in wireless communications. All of these values are summarized in Table VI. For each of the fixed schemes the data was interleaved over four consecutive TDD frames, yielding a latency of about $30 \mathrm{~ms}$.

The FER was evaluated for the four fixed schemes shown in Table VI through Rayleigh fading channels for normalized Doppler frequencies of 0.0042, 0.025, 0.054, 0.079, and 0.133, which correspond to mobile velocities of $0.136,0.812,1.75$, 2.57, and $4.32 \mathrm{~ms}^{-1}$. A minimum of 10000 frames were simulated. The FER's were evaluated with and without interleaving and the corresponding SNR values where the $1 \%$ FER was achieved was recorded. The SNR's required for the lowest and highest normalized Doppler frequencies are shown in Table VII. Under slow fading conditions the interleaving had no effect upon the required average SNR, however, for the fastest fading it resulted in a reduction of $2.6-4.3 \mathrm{~dB}$ in terms of the required average channel SNR.

\section{B. Adaptive Scheme}

In adaptive modulation, a frame error can occur for two reasons, either as a result of the noise corrupting a sufficient 
TABLE VIII

Transmission Rate for Statisticlly Multiplexed Adaptive Modulation Scheme Showing the Desired Number of Slots to Achieve a Throughrut Comparable with the Fixed Scheme in Table VI

\begin{tabular}{c|c|c|c|c}
\hline $\begin{array}{c}\text { Current } \\
\text { Transmission } \\
\text { Mode }\end{array}$ & $\begin{array}{c}\text { Codewords } \\
\text { Per } \\
\text { Slot }\end{array}$ & $\begin{array}{c}\text { Information } \\
\text { Bits Per } \\
\text { Slot }\end{array}$ & $\begin{array}{c}\text { Desired } \\
\text { Multiplexed } \\
\text { Slots }\end{array}$ & $\begin{array}{c}\text { Info } \\
\text { Per } \\
\text { Frame }\end{array}$ \\
\hline No Transmission & 0 & 0 & 1 & 0 \\
BPSK & 1 & 39 & 5 & 195 \\
QPSK & 2 & 78 & 2.5 & 195 \\
QAM 16 & 4 & 156 & 1.25 & 195 \\
QAM 64 & 6 & 234 & $0.8 \dot{3}$ & 195 \\
\hline
\end{tabular}

number of bits, such that the $\mathrm{BCH}$ codeword is overloaded, or, alternatively the frame experiences too high a delay and it is therefore not transmitted. The frames lost due to corruption yield the FER, the frames lost because of delay result in a delay error rate (DER) and the total rate of corrupted frames is the total error rate (TER). For the fixed schemes the DER was zero and therefore TER equals FER.

An ideal adaptive modulation scheme balances the DER and FER in order to minimize the TER and this is to a certain extent analogous to the tradeoff in BER and BPS performance. From the frequency hopping investigation in Section VI, it is clear that DER can be reduced for no cost in FER. However, the intention of this comparative study is to employ adaptive modulation under the most demanding conditions and therefore a single-frequency statistical multiplexing is used to reduce the DER without frequency hopping.

\section{Performance Evaluation}

The switching SNR's to be used for the adaptive modulation scheme were the speech system's optimized mean BER levels and the speech system's peak BER levels from Table I. We opted for employing the block code $\mathrm{BCH}(63,39,4)$, since it was the most robust code from the $n=63$-b-long $\mathrm{BCH}$ code family that would achieve the $41.6 \mathrm{~Kb} / \mathrm{s}$ useful target rate without the required $\Xi$ value becoming too high for average channel SNR's of approximately $20 \mathrm{~dB}$. Depending upon which mode the adaptive modem was in, $0,1,2,4$, or 6 codewords could be transmitted per frame as exemplified by Table VIII.

The performance of the adaptive scheme was evaluated using the statistical multiplexing algorithm from Section VI. The ratio $\Xi$ was no longer fixed. Here, the adaptive modulation and statistical multiplexing support $195 \mathrm{~b}$ /frame for every user that is accommodated and therefore, irrespective of the average channel SNR, each user adds five $\operatorname{BCH}(63,39,4)$ codewords to its queue. This yields a throughput of 42.25 $\mathrm{Kb} / \mathrm{s}$, which is slightly in excess of the target value of 41.6 $\mathrm{Kb} / \mathrm{s}$. Frames that were in the transmission buffer longer than $30 \mathrm{~ms}$ were removed from the buffer and were not transmitted. The relative frequency of this event gave the above-mentioned delay-induced DER estimate. Perfectly coherent detection and channel estimation was assumed, as it had been for the fixed modulation, and the SNR was assumed to be constant over a $72-\mu$ s slot. The FER was evaluated using the same technique as was used for fixed modulation and the results were obtained for the same slow and fast fading rates. All users transmitting in the TDD frame had the same throughput requirements, average channel SNR and fading statistics. Table IX shows the DER, FER, and TER for both the mean BER and peak BER switching SNR's of Table I, in both the slow and fast Rayleigh fading channels, for various numbers of users at average channel SNR's when all the users are generally achieving the desired error rate and delay performance.

Several observations may be made from Table IX.

- The DER with peak BER switching SNR's is higher than with mean BER switching SNR's. This is because the peak BER switching SNR's are higher, and, therefore, the throughput is on average lower. This means that there is more possibility of frames being dropped from the transmission buffer due to delayed transmission.

- The FER with peak BER switching SNR's is less than with mean BER switching SNR's. This is the reverse situation compared to above, since the higher switching SNR's result in higher integrity for the data transfer.

- The FER is the same for the equivalent fast and slow fading scenarios, however, as expected, the DER reduces with increased mobile speed.

- When the number of users increases, while maintaining the same average channel SNR, the DER increases. This is a result of the extra contention for slots when using low-order modem modes.

- When the average channel SNR increases, while maintaining the same number of users, the DER reduces. This is because all users will on average require fewer slots or experience fewer "no transmission" instances.

From the table it can be concluded that combined adaptive modulation and statistical multiplexing can support 5, 10, $15,20,25$, or 30 TDD users at 20-, 20-, 22-, 26-, 28-, and 30-dB average channel SNR's, respectively, at either of the investigated velocities or at either set of switching SNR's. The number of users supported by the fixed modulation schemes can be calculated by taking the 32 duplex slot pairs and dividing them by the number of slots required per frame, as given in Table VI. The results are shown in Fig. 8 for the fast and slow fading environments, with and without interleaving, although the points for the slow channel coincide. The adaptive performance is also shown, however, it should be noticed that the line given is for the slow fading channel and better performance is achieved by the fast fading channel, hence, the label "worst case."

Fig. 8 shows that the adaptive scheme can support at least as many, and often $25 \%$ more multimedia users than the BPSK, QPSK, and square 16 fixed modulation schemes over the worst case slow fading channel. Through the fast fading channel, with the exception of 5 users, the adaptive modulation can match the performance all the fixed schemes but at slightly lower average channel SNR's. There are however, two key points that have not been expressed so far, which further improve the performance of adaptive modulation over the fixed schemes that are elaborated on below.

- The overall effect of a given DER and FER upon the quality of a wireless service is not the same. With a 
TABLE IX

The Frame Error Rate (FER) Due to Noise, Dropped Error Rate (DER), and Total Error Rate (TER) for Statistically Multiplexed Block-Coded Adaptive Modulation Scheme Using the Peak and Mean BeR Switching SNR's at Two Velocities

\begin{tabular}{|c|c|c|c|c|c|c|c|c|c|c|c|c|c|}
\hline \multirow{3}{*}{$\begin{array}{l} \\
\text { Users }\end{array}$} & \multirow[b]{3}{*}{ SNR $(\mathrm{dB})$} & \multicolumn{6}{|c|}{ Normalised Doppler Frequency 0.0042 (velocity $0.136 \mathrm{mss}^{-1}$ ) } & \multicolumn{6}{|c|}{ Normalised Doppler Frequency 0.133 (velocity $4.32 \mathrm{~ms}^{-1}$ ) } \\
\hline & & \multicolumn{3}{|c|}{ Mean BER switching SNRs } & \multicolumn{3}{|c|}{ Peak BËR switching SNRs } & \multicolumn{3}{|c|}{ Mean BERR switching SNRs } & \multicolumn{3}{|c|}{ Peak BER switching SNRs } \\
\hline & & FER & DER & TËR & FER & DER & THR. & FEK & DER & TER & FER & DER & $\mathrm{T} \mathrm{T} \mathrm{ER}$ \\
\hline 5 & 18 & $3.0 \times 10^{-3}$ & $3.5 \times 10^{-3}$ & $1.3 \times 10^{-2}$ & $4.4 \times 10^{-5}$ & $1.4 \times 10^{-2}$ & $1.4 \times 10^{-2}$ & $3.0 \times 10^{-3}$ & $1.8 \times 10^{-3}$ & $4.8 \times 10^{-3}$ & $4.4 \times 10^{-5}$ & $2.9 \times 10^{-3}$ & $2.9 \times 10^{-3}$ \\
\hline 5 & 20 & $2.3 \times 10^{-3}$ & $5.8 \times 10^{-3}$ & $8.1 \times 10^{-3}$ & $4.1 \times 10^{-5}$ & $7.2 \times 10^{-3}$ & $7.2 \times 10^{-3}$ & $2.3 \times 10^{-3}$ & $1.0 \times 10^{-3}$ & $3.3 \times 10^{-3}$ & $4.1 \times 10^{-5}$ & $1.4 \times 10^{-3}$ & $1.4 \times 10^{-3}$ \\
\hline 10 & 20 & $2.3 \times 10^{-3}$ & $6.1 \times 10^{-3}$ & $8.4 \times 10^{-3}$ & $4.1 \times 10^{-5}$ & $7.6 \times 10^{-3}$ & $7.6 \times 10^{-3}$ & $2.3 \times 10^{-3}$ & $1.1 \times 10^{-3}$ & $3.4 \times 10^{-3}$ & $4.1 \times 10^{-5}$ & $3.4 \times 10^{-3}$ & $3.4 \times 10^{-3}$ \\
\hline 10 & 22 & $1.7 \times 10^{-3}$ & $2.0 \times 10^{-3}$ & $3.7 \times 10^{-3}$ & $3.3 \times 10^{-5}$ & $3.4 \times 10^{-3}$ & $3.4 \times 10^{-3}$ & $1.7 \times 10^{-3}$ & $2.2 \times 10^{-4}$ & $1.9 \times 10^{-3}$ & $3.3 \times 10^{-5}$ & $3.7 \times 10^{-4}$ & $4.0 \times 10^{-4}$ \\
\hline 15 & 22 & $1.7 \times 10^{-3}$ & $3.0 \times 10^{-3}$ & $4.7 \times 10^{-3}$ & $3.3 \times 10^{-5}$ & $5.5 \times 10^{-3}$ & $5.5 \times 10^{-3}$ & $1.7 \times 10^{-3}$ & $2.9 \times 10^{-4}$ & $2.0 \times 10^{-3}$ & $3.4 \times 10^{-5}$ & $4.1 \times 10^{-4}$ & $4.4 \times 10^{-4}$ \\
\hline 15 & 24 & $1.2 \times 10^{-3}$ & $9.3 \times 10^{-4}$ & $2.1 \times 10^{-3}$ & $2.4 \times 10^{-5}$ & $1.5 \times 10^{-3}$ & $1.5 \times 10^{-3}$ & $1.2 \times 10^{-3}$ & $6.7 \times 10^{-5}$ & $1.3 \times 10^{-3}$ & $2.4 \times 10^{-5}$ & $1.0 \times 10^{-4}$ & $1.2 \times 10^{-4}$ \\
\hline 20 & 26 & $8.7 \times 10^{-4}$ & $1.1 \times 10^{-3}$ & $2.0 \times 10^{-3}$ & $1.7 \times 10^{-5}$ & $1.7 \times 10^{-3}$ & $1.7 \times 10^{-3}$ & $9.0 \times 10^{-4}$ & $7.3 \times 10^{-5}$ & $9.7 \times 10^{-4}$ & $1.7 \times 10^{-5}$ & $1.0 \times 10^{-4}$ & $1.2 \times 10^{-4}$ \\
\hline 20 & 28 & $4.6 \times 10^{-4}$ & $6.6 \times 10^{-4}$ & $1.1 \times 10^{-3}$ & $1.2 \times 10^{-5}$ & $1.0 \times 10^{-3}$ & $1.0 \times 10^{-3}$ & $4.8 \times 10^{-4}$ & $3.5 \times 10^{-5}$ & $5.2 \times 10^{-4}$ & $1.2 \times 10^{-5}$ & $7.4 \times 10^{-5}$ & $8.6 \times 10^{-5}$ \\
\hline 25 & 28 & $4.6 \times 10^{-4}$ & $8.6 \times 10^{-4}$ & $1.3 \times 10^{-3}$ & $1.1 \times 10^{-5}$ & $8.4 \times 10^{-3}$ & $8.4 \times 10^{-3}$ & $4.7 \times 10^{-4}$ & $2.5 \times 10^{-4}$ & $7.2 \times 10^{-4}$ & $1.2 \times 10^{-5}$ & $3.7 \times 10^{-1}$ & $3.8 \times 10^{4}$ \\
\hline 25 & 30 & $3.8 \times 10^{-4}$ & $5.4 \times 10^{-4}$ & $9.2 \times 10^{-4}$ & $7.0 \times 10^{-6}$ & $6.2 \times 10^{-3}$ & $6.2 \times 10^{-3}$ & $3.8 \times 10^{-4}$ & $2.1 \times 10^{-5}$ & $4.0 \times 10^{-4}$ & $7.0 \times 10^{-6}$ & $3.6 \times 10^{-5}$ & $1.3 \times 10$ \\
\hline 30 & 3 & $4.0 \times 10^{-4}$ & $2.0 \times 10^{-3}$ & $2.4 \times 10^{-3}$ & $7.0 \times 10^{-6}$ & $1.2 \times 10^{-2}$ & $1.2 \times 10^{-2}$ & $4.1 \times 10^{-4}$ & $1.8 \times 10^{-3}$ & $2.2 \times 10^{-3}$ & $8.0 \times 10^{-6}$ & $4.4 \times 10^{3}$ & $4.4 \times 10^{-3}$ \\
\hline 30 & 32 & $2.4 \times 10^{-4}$ & $6.1 \times 10^{-4}$ & $8.8 \times 10^{-4}$ & $6.0 \times 10^{-6}$ & $3.1 \times 10^{-3}$ & $3.1 \times 10^{-3}$ & $2.5 \times 10^{-4}$ & $4.1 \times 10^{-5}$ & $2.9 \times 10^{-4}$ & $6.0 \times 10^{-6}$ & $4.8 \times 10^{-5}$ & $5.4 \times 10^{-5}$ \\
\hline
\end{tabular}

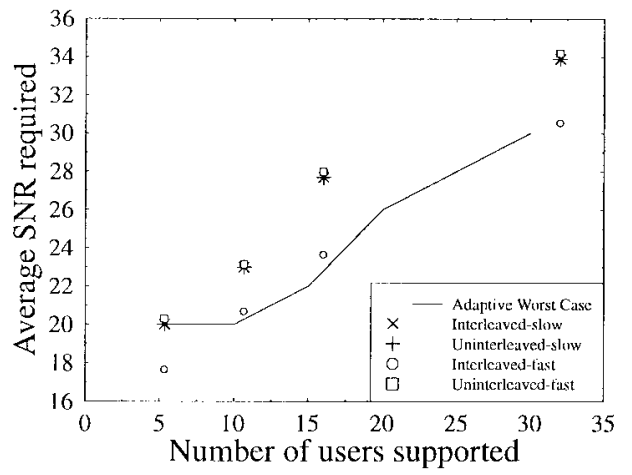

Fig. 8. The average channel SNR required to support a given number of users with fixed and adaptive modulation on the basis of the comparative study. The adaptive performance is shown for the "slow" fading channel, and it is hence labeled as "worst case."

fixed scheme the DER is zero, and therefore all errors are caused by corruption, rather than delay. This has the advantage that since systematic block codes are used, the decoder can output the received information bits, rather than erroneously decoding them when the code is overloaded. Alternatively, the overloaded block may be ignored at the receiver. Either way, without the use of automatic repeat request (ARQ) techniques, which exhibit an associated delay, redundancy, and additional complexity, the transmitting source encoder does not know the condition of the received block. With adaptive modulation and the peak switching SNR's the FER can become very low, as seen in Table IX, and the DER approximates the TER. As a consequence of this, assuming an FER $=0$ and with the knowledge of when packets are dropped, it possible for the source encoder to have nearperfect knowledge of what is received at the decoder. This is useful in preventing the propagation of errors resulting from the encoder ignorantly assuming that its local decoded state is the same as the decoded state at the other end of the link.

- The results for the fixed modulation were evaluated using BPSK, QPSK, square 16, and 64 QAM. The optimum operating SNR range of these fixed schemes is based around a particular SNR value. If the average channel SNR increases, the BER performance will improve, and if the average channel SNR reduces the BER performance will deteriorate. There is no capacity for reassigning the number of slots to a user, as the average channel SNR varies. Without this reassignment every fixed modulation user will potentially require a margin of SNR to protect it against reducing average channel SNR's. Furthermore, as the average channel SNR increases, the lack of capacity to reassign slots results in fewer users being supported than possible. Slot reassignment is possible for the fixed modulation schemes [41], however, it incurs an additional transceiver reconfiguration capability beyond the scope of this work.

Fig. 8 shows that adaptive modulation increases the number of users that can be supported at a given average channel SNR over a Rayleigh fading channel when compared with fixed modulation. Although all of the points in Fig. 8 achieve the TER of $1 \%$, the illustration does not show by what margin this target is achieved. To rectify this, Fig. 9 considers the TER for the adaptive and fixed modulation schemes from Fig. 8. The number of users is, therefore, a variable in Fig. 9, however, the number of users supported by the adaptive schemes is always equal or greater than the number of users supported by the fixed schemes, as was shown in Fig. 8.

Considering Fig. 9(a) it can be seen for the slow fading channel that with the exception of one value the adaptive scheme results in a higher integrity in terms of TER than the fixed scheme. Furthermore, for the slow fading channel there is no clear advantage in using either the peak or mean BER adaptive switching SNR's. At the higher speed seen in Fig. 9(b), both sets of switching SNR's show even greater improvements over the fixed schemes and there is a marked advantage in using the peak BER switching SNR's. The mean and peak switching SNR's result, respectively, in up to a factor of three or 30 reduction in TER, respectively. The peak BER switching SNR's are preferable for the faster fading channel to the mean BER switching SNR's because the DER becomes negligible for both sets of switching thresholds, as the channel correlation is reduced. Therefore, the TER tends to the FER, which is much lower for the peak BER switching SNR's.

The net result of Figs. 8 and 9 is that adaptive modulation improves the quality of the link expressed in terms of TER, while accommodating additional users. In conclusion, we note 


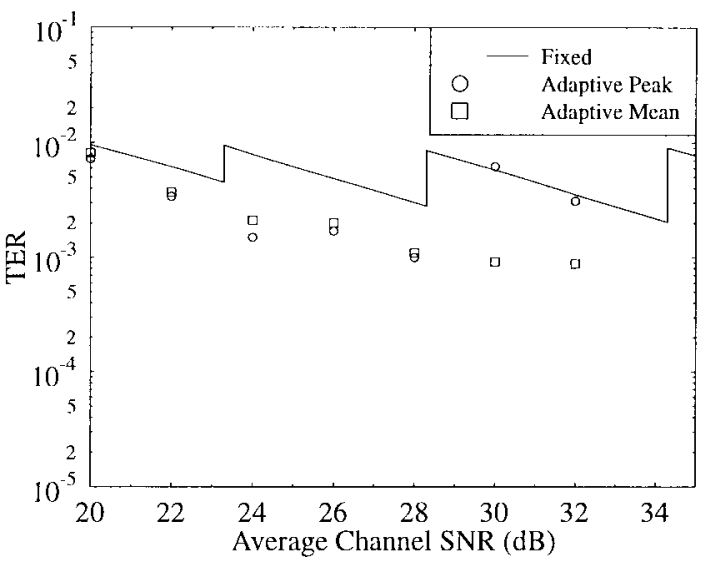

(a)

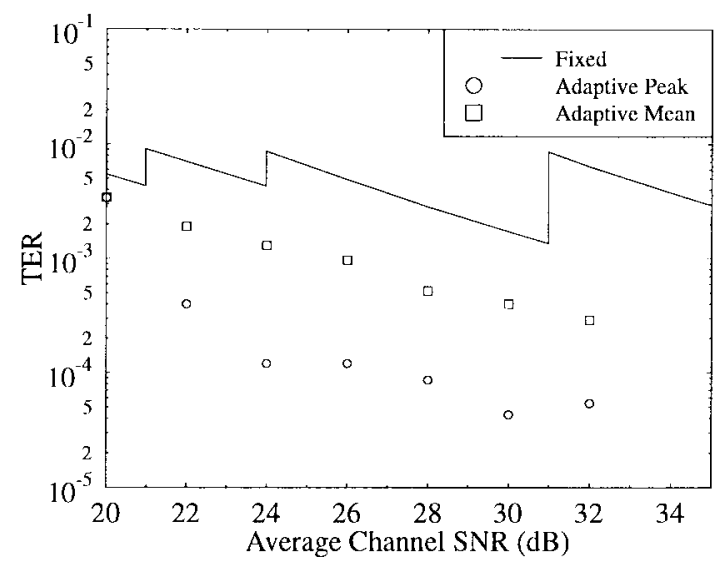

(b)

Fig. 9. Total error rate for the adaptive and interleaved fixed modulation schemes at normalized Doppler frequencies of (a) 0.004170 (slow) and (b) 0.133427 (fast). The adaptive schemes are shown with both mean and peak BER switching SNR's and the number of users that are supported can be inferred from Fig. 8.

that in this section the system's performance was studied under the assumption of a practical third-generation system framework, which may appear specific at first sight. Nonetheless, the findings of this paper are quite generic, since the most critical system parameters are the frame and slot duration in terms of the system's efficiency and the values affecting our system's performance were quite similar to those of other existing standard systems, such as the GSM system [32] or the DECT system [27]. Let us finally conclude our discourse on the latency aspects of adaptive modulation.

\section{CONCLUSIONS}

Previous work has shown that adaptive modulation can mitigate the effects of fading and maintain an arbitrary target mean BER. In this paper, a Markov model has been used to simulate the varying states of an adaptive modem. Two techniques for generating the model were considered, that proposed by Wang and Moayeri [38], and a more empirical approach, which was able to model nonadjacent modem mode transitions encountered under faster fading conditions. It was concluded that the latter technique was more suitable for higher normalized Doppler frequencies.

A buffer was proposed between the source/channel encoder and the modulator, in order to accommodate the variation in throughput induced by the proposed adaptive scheme. This introduced latency and we considered, how the latency varied with the normalized Doppler frequency and average channel SNR in Figs. 4 and 5, for various values of the relative channel utilization factor $\Xi$. It was also demonstrated in Fig. 6 that frequency hopping can be employed to overcome this latency. Then a more sophisticated technique, using statistical multiplexing was proposed. The results of statistical multiplexing were presented in Fig. 7. These results identified the optimum contention permission probability $p$ for our considered scenario to be around 0.3 .

All in all, adaptive modulation has a strong case to be used for computer data, where data integrity is much more important than latency. Without losing sight of that, a case study of adaptive modulation versus fixed modulation in a lowdelay, interactive mobile multimedia scenario was considered in Section VII. The fixed modulation and $\mathrm{BCH}$ error correction schemes characterized in Table VI were compared with the adaptive, statistical multiplexing-assisted scheme summarized in Table VIII. The comparison identified that adaptive modulation and statistical multiplexing may increase the number of users in a system by $25 \%$ without an increase in average channel SNR, which is supported by Fig. 8. Furthermore, the quality of service in terms of the TER offered to the users would be increased by a maximum of a factor of 30, as shown in Fig. 9. These features provide further justification for the inclusion of adaptive modulation within a multimode UMTS air interface toolbox. Our future work is concentrated on interference cancellation, channel coding, power control, and equalization for adaptive modems.

\section{APPENDIX \\ STATISTICAL MULTIPLEXING IMPLEMENTATION}

All mobiles are aware of which slots are allocated to which user on the basis of the broadcast information contained in the control slots of Fig. 2. Without statistical multiplexing a slot is allocated to a user unconditionally. Therefore, if the user cannot completely fill that slot with information bits it is still worth transmitting the few information bits that are in the buffer along with some dummy bits. However, with statistical multiplexing a slot only partially filled with information bits by one user could have been completely filled with information bits by another user. Therefore, in the statistical multiplexing experiments a slot that would have only partially be filled with information bits is not transmitted and the bits are queued for the next frame.

Fig. 10 illustrates the operation of the base station for employing statistical multiplexing. The processes in the flowchart are now elaborated upon.

1) Control Slots: The base station occupies the first two of the 64 slots for transmitting broadcast messages. These 


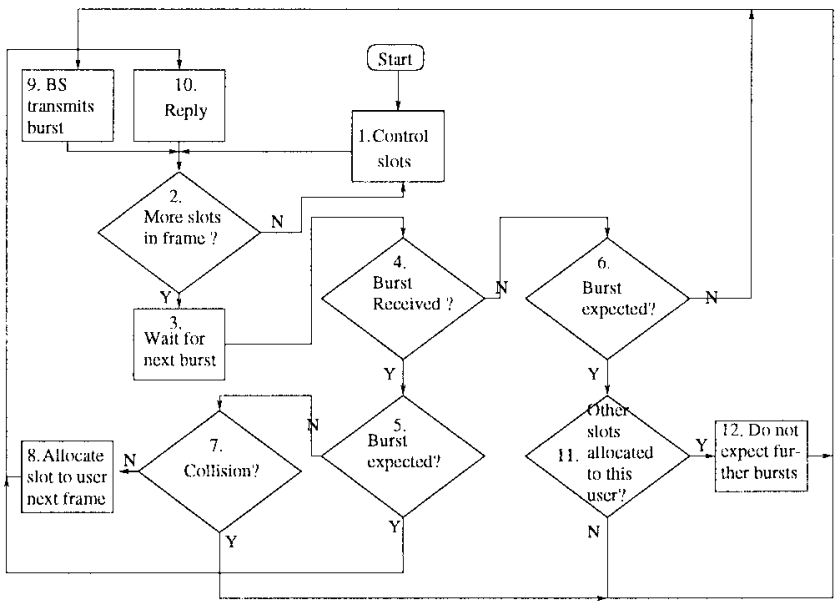

Fig. 10. The base-station implementation of statistical multiplexing algorithm.

slots inform each user as to which slots are allocated to them on the basis of their requirements in the previous frame. All users receive these slots and it is assumed that they are received without errors. Again, the slots are allocated such that for each user all of the pairs of uplink and downlink slots in a particular frame are next to each another.

2) More Slots in Frame?: If there are more slots in the frame (i.e., the last slot transmitted was not number 64), which have not been considered so far, then they must be processed, otherwise, after considering the last slot in the TDD frame the base station transmits another pair of broadcast control slots.

3) Wait for Next Burst: The base station waits for a received burst.

4) Burst Received?: The base station determines whether a burst was transmitted by any mobile.

5) Burst Expected?: If any mobile did transmit in the slot, was it the mobile that was allocated that slot in the control slots? An unexpected mobile transmission could only happen if the slot concerned was not allocated to a specific user in the broadcast control slot. This, of course, is on the premise that the control slot is successfully received.

6) Burst Expected?: If no mobile transmitted in the slot, was the slot allocated to a particular user? This would be the case, if a slot had been allocated to a user, but that user did not have a sufficient number of bits in the buffer in order to render their transmission worthwhile. Alternatively, the channel quality was deemed so low that the mobile decided not to jeopardize degrading the BER performance by transmitting.

7) Collision?: If simultaneously more than one burst was received, then the received energy was typically high, but the data was corrupted, which was the result of a packet collision condition.

8) Allocate Slot to User: This slot is now allocated to the user in question for future frames.

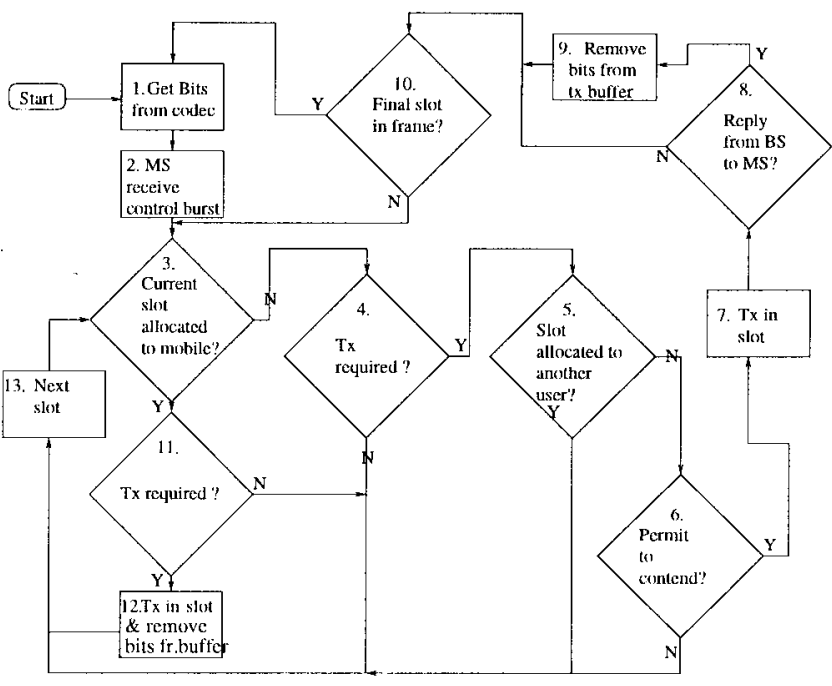

Fig. 11. The mobile station's statistical multiplexing algorithm.

9) BS Transmits Burst: No users have used the previous uplink slot, hence the base station transmits a burst as a "beacon" for any users, who wish to transmit in the next slot in order to obtain a measure of the channel quality.

10) Reply: One and only one user has transmitted in the previous uplink slot. On the basis of the channel quality the base station replies using the appropriate modulation scheme. All users, who were permitted to transmit in the next uplink slot, will "passively receive" this downlink burst in order to estimate the channel quality.

11) Other Slots Allocated to This User?: Were there any slots in positions after the current one in the frame allocated to the user who did not transmit in this one?

12) Do Not Expect Further Bursts: Other slots allocated to the user in the frame will be available for other users in this and following frames.

Having considered the BS's actions, Fig. 11 illustrates the operation of the mobile station for using statistical multiplexing. The processes in the flowchart are to a certain extent similar to those of the BS's and hence for space economy we refrain from guiding the reader through it.

Below it will be demonstrated that the proposed statistical multiplexing scheme will result in reduced latency. This will allow the system throughput to be close to the mean theoretical BPS performance. This is equivalent to saying that $\Xi$ may be increased without introducing unacceptable delay. An alternative view is that statistical multiplexing mitigates the varying throughput that results from adaptive modulation. However, statistical multiplexing will result in a variable expected BER of a frame around the mean target BER. This scenario is exemplified by the situation, where the fading is extremely slow and transmission of several frames takes place when the SNR is either slightly above, or below, a switching SNR. Specifically, if the SNR is slightly above a switching SNR, then an intolerable burst of errors may be experienced. Therefore, the peak BER switching SNR's that were portrayed in Table I were used in the associated experiments. 


\section{ACKNOWLEDGMENT}

The helpful corrections of the anonymous reviewer are gratefully acknowledged by the authors.

\section{REFERENCES}

[1] R. Steele and W. T. Webb, "Variable rate QAM for data transmission over Rayleigh fading channels," in Wireless'91, Calgary, Alta., Canada, pp. $1-14$.

[2] W. Webb and R. Steele, "Variable rate QAM for mobile radio," IEEE Trans. Commun., vol. 43, pp. 2223-2230, July 1995.

[3] W. T. Webb and L. Hanzo, Modern Quadrature Amplitude Modulation: Principles and Applications for Fixed and Wireless Channels. New York: IEEE Press, 1994, p. 557.

[4] Y. Kamio, S. Sampei, H. Sasaoka, and N. Morinaga, "Performance of modulation-level-control adaptive-modulation under limited transmission delay time for land mobile communications," in 45th Vehicular Technology Conf., 1995, pp. 221-225.

[5] S. Sampei, S. Komaki, and N. Morinaga, "Adaptive modulation/TDMA scheme for large capacity personal multi-media communication systems," IEICE Trans. Commun., vol. 77, no. 9, pp. 1096-1103, 1994.

[6] M. Morimoto, H. Harada, M. Okada, and S. Komaki, "A study on power assignment of hierarchical modulation schemes for digital broadcasting," IEICE Trans. Commun., vol. 77, no. 12, pp. 1495-1500, 1994.

[7] S. Otsuki, S. Sampei, and N. Morinaga, "Square-QAM adaptive modulation TDMA/TDD systems using modulation level estimation with Walsh function," Electron. Lett., pp. 169-171, Nov. 1995.

[8] S. Sampei, T. Ue, N. Morinaga, and K. Hamguchi, "Laboratory experimental results of an adaptive modulation TDMA/TDD for wireless multimedia communication systems," in Proc. IEEE PIMRC'97, Helsinki, Finland, Sept. 1-4, 1997, pp. 467-471.

[9] K. Arimochi, S. Sampei, and N. Morinaga, "Adaptive modulation system with discrete power control and predistortion-type nonlinear compensation for high spectral efficient and high-power efficient wireless communication systems," in Proc. IEEE PIMRC'97, Helsinki, Finland, Sept. 1-4, 1997, pp. 472-477.

[10] M. Naijoh, S. Sampei, N. Morinaga, and Y. Kamio, "ARQ schemes with adaptive modulation/TDMA/TDD systems for wireless multimedia communication systems," in Proc. IEEE PIMRC'97, Helsinki, Finland, Sept. 1-4, 1997, pp. 709-713.

[11] S.-G. Chua and A. Goldsmith, "Variable-rate variable-power MQAM for fading channels," in 46th Vehicular Technology Conf., 1996, pp. $815-819$.

[12] A. Goldsmith and S. Chua, "Variable-rate variable-power MQAM for fading channels," IEEE Trans. Veh. Technol., to be published.

[13] A. Goldsmith, "The capacity of downlink fading channels with variable rate and power," IEEE Trans. Veh. Technol., vol. 46, pp. 569-580, Aug. 1997.

[14] A. Goldsmith and P. Varaiya, "Capacity of fading channels with channel side information," IEEE Trans. Inform. Theory, to be published.

[15] M.-S. Alouini and A. Goldsmith, "Capacity of fading channels under different adaptive transmission and diversity techniques," IEEE Trans. Veh. Technol., to be published.

[16] _ "Area spectral efficiency of cellular mobile radio systems," IEEE Trans. Veh. Technol., to be published.

[17] A. Goldsmith and S. Chua, "Adaptive coded modulation," IEEE Trans. Commun., to be published.

[18] D. A. Pearce, A. G. Burr, and T. C. Tozer, "Comparison of countermeasures against slow Rayleigh fading for TDMA systems," in Colloquium on Advanced TDMA Techniques and Applications, 1996, pp. $9 / 1-9 / 6$.

[19] J. M. Torrance and L. Hanzo, "Upper bound performance of adaptive modulation in a slow Rayleigh fading channel," Electron. Lett., pp. 169-171, Apr. 1996

[20] _ "Adaptive modulation in a slow Rayleigh fading channel," in Proc. 7th Personal, Indoor and Mobile Radio Communications (PIMRC) Conf., 1996, pp. 497-501.

[21] _ "Demodulation level selection in adaptive modulation," Electron. Lett., vol. 32, no. 19, pp. 1751-1752, Sept. 1996.

[22] _ "Latency considerations for adaptive modulation in slow Rayleigh fading," in Proc. IEEE VTC'97, Phoenix, AZ, 1997, pp. 1204-1209.

[23] _ " "Optimization of switching levels for adaptive modulation in a slow Rayleigh fading channel," Electron. Lett., pp. 1167-1169, June 1996.
[24] W. H. Press, S. A. Teukolsky, W. T. Vetterling, and B. P. Flannery, Numerical Recipes in C. Cambridge, U.K.: Cambridge Univ. Press, 1994.

[25] D. Greenwood and L. Hanzo, Mobile Radio Channels, R. Steele, Ed. London, U.K.: Pentech, 1992, ch. 2

[26] A. Papoulis, Probability Random Variables, and Stochastic Processes, 3rd ed. New York: McGraw-Hill, 1991.

[27] "Digital European cordless telecommunications interface-Part 2: Physical layer," pp. 175-172, 1992.

[28] B. Friedrichs, "Report to FIRST partners on ATDMA proposal," Bosch Telecom GmbH Tech. Rep., 1996.

[29] R. Karimi, "Report to FIRST partners: FRAMES air-interface," Motorola GDP Research Group Tech. Rep., 1996.

[30] F. de Ryck, H. Persson, T. Leskinen, T. Ojanpera, and W. Mohr "FRAMES contribution to the standardization of the UMTS air interface," in ACTS Mobile Telecommunications Summit, Nov. 27-29, 1996, pp. 244-249.

[31] "Groupe speciale mobile (GSM) recommendation," ETSI, Apr. 1988.

[32] L. Hanzo and J. Stefanov, "The Pan-European digital cellular mobile radio system-known as GSM," in Mobile Radio Communications, 2nd ed., R. Steele, Ed. London, U.K.: Pentech, 1994, ch. 8, pp. 677-773.

[33] W. C. Jakes, Microwave Mobile Communications. New York: Wiley, 1974.

[34] E. Casas and C. Leung, "A simple digital fading simulator for mobile radio," IEEE Trans. Veh. Technol., vol. 39, no. 3, pp. 205-212, 1990

[35] G. A. Arredondo, W. H. Chriss, and E. H. Walker, "A multipath fading simulator for mobile radio," IEEE Trans. Veh. Technol., vol. VT-22, no. 4, pp. 241-244, 1973

[36] R. A. Goubran, H. M. Hafez, and A. U. H. Sheikh, "Real-time programmable land mobile channel simulator," in 36th IEEE Vehicular Technology Conf., 1986, pp. 215-218.

[37] R. B. D'Agostino and M. A. Stephens, Eds., Goodness-of-Fit Techniques. New York: Marcel Dekker, 1986.

[38] H. Wang and N. Moayeri, "Finite-state Markov channel-A useful model for radio communication channels," IEEE Trans. Veh. Technol., vol. 44, no. 1, pp. 163-171, 1995.

[39] R. A. Salami et al., "Speech coding," in Mobile Radio Communications, 2nd ed., R. Steele, Ed. London, U.K.: Pentech, 1994, ch. 3, pp. $186-346$.

[40] J. P. Woodard, J. M. Torrance, and L. Hanzo, "A low delay multimode speech terminal," in 46th Vehicular Technology Conf., 1996, pp. 213-217.

[41] J. Williams, L. Hanzo, and R. Steele, "Channel adaptive modulation," in Proc. IEE Conf. Radio Receivers and Associated Systems, 1995, pp. 144-147.

[42] D. J. Goodman, R. A. Valuenzuela, K. T. Gayliard, and B. Ramamurthi, "Packet reservation multiple access for local wireless communications," IEEE Trans. Commun., vol. 37, pp. 885-890, 1989.

[43] D. J. Goodman and S. X. Wei, "Efficiency of packet reservation multiple access," IEEE Trans. Veh. Technol., vol. 40, no. 1, pp. 170-176, 1991.

[44] S. Nanda, D. J. Goodman, and U. Timor, "Performance of PRMA: A packet voice protocol for cellular systems," IEEE Trans. Veh. Technol., vol. 40, no. 3, pp. 584-598, 1991.

[45] W.-C. Wong, "Dynamic allocation of packet reservation multiple access carriers," IEEE Trans. Veh. Technol., vol. 42, no. 4, pp. 385-392, 1993.

[46] "Recommendation G. 729: Coding of speech at $8 \mathrm{kbits} / \mathrm{s}$ using conjugate-structure algebraic-code-excited-linear-prediction (CSACELP)," ITU-T Tech. Rep., 1996.

[47] "Recommendation H. 263: Video coding for low bitrate communications," ITU-T Tech. Rep., 1996.

[48] K. H. H. Wong and L. Hanzo, "Channel coding," in Mobile Radio Communications, R. Steele, Ed. London, U.K.: Pentech, 1992, ch. 4, pp. 347-489.

Jeff M. Torrance received the Ph.D. degree in digital modulation from the University of Southampton, Southampton, U.K.

In 1997, he joined the Wireless Technology Practice at PA Consulting Group, Cambridge, U.K. He is currently responsible for a team working on third-generation mobile communications developments. 
Lajos Hanzo received the Ph.D. degree in 1983 from the Technical University of Budapest, Hungary.

During his 20-year career in telecommunications, he has held various research and academic posts in Hungary, Germany, and the U.K. Since 1986 , he has been with the Department of Electronics and Computer Science, University of Southampton, Southampton, U.K. He is also a consultant to Multiple Access Communications Ltd., U.K. He coauthored three books on mobile radio communications, published more than 200 research papers, organized and chaired conference sessions, presented overview lectures, and was awarded a number of distinctions. Currently, he is managing a research team, working on a range of research projects in the field of wireless multimedia communications under the auspices of the Engineering and Physical Sciences Research Council (EPSRC), U.K., the European Advanced Communications Technologies and Services (ACTS) Programme, and the Mobile Virtual Centre of Excellence (VCE), U.K. He also is a Chair in Telecommunications. As an enthusiastic supporter of industry-academia liaisons, he offers a range of short courses on all aspects of wireless multimedia communications. Further information on research in progress and associated publications can be found at http://www-mobile.ecs.soton.ac.uk. 